\title{
Fare la storia del secondo Novecento con le canzoni italiane
}

\author{
di Aldo Gianluigi Salassa
}

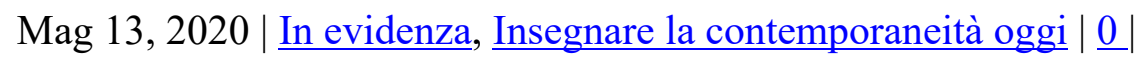

\section{5 laboratori proposti da Istoreto}

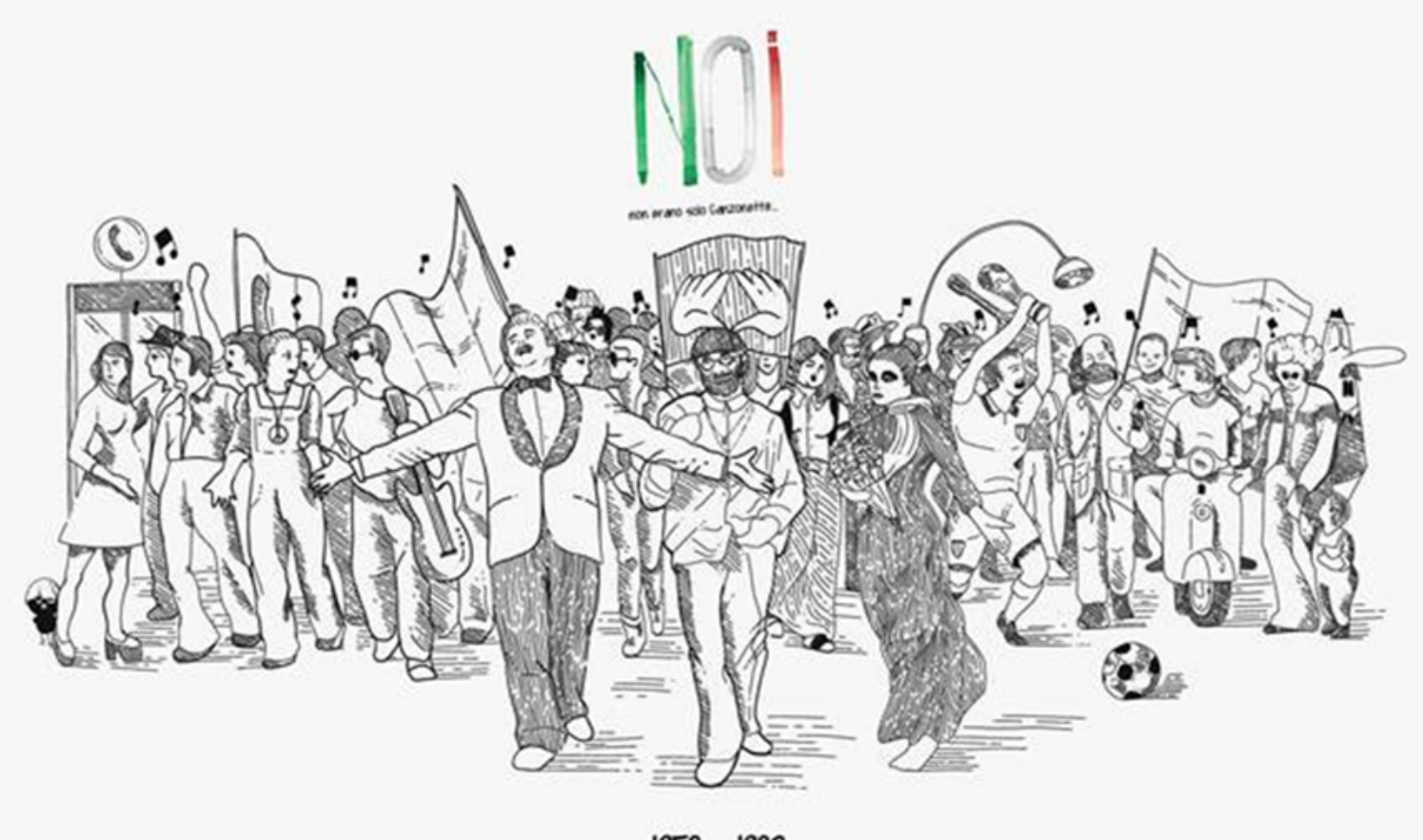

$1958-1982$

Il manifesto artistico della mostra

\begin{abstract}
A partire da un evento pubblico come una mostra strutturata sulla canzone d'autore degli anni Sessanta e Settanta, alcuni laboratori, costruiti specificatamente nello spazio espositivo, hanno permesso di approfondire con gli studenti gli aspetti più importanti della trasformazione della società italiana di allora.
\end{abstract}

\section{Un'attività collegata a una mostra}

Nel corso del 2019 l'Istituto piemontese per la storia della Resistenza e della società contemporanea "Giorgio Agosti" ha proposto alle scuole secondarie una serie di laboratori, collegati alla mostra NOI... non erano solo canzonette allestita alla Promotrice delle Belle Arti di Torino. La mostra attualmente a Bologna (Palazzo Belloni) fino ad aprile 2020[1] - è una produzione di Bibibus Events, a cura di Gianpaolo Brusini, Giovanni De Luna, Lucio Salvini e con la partecipazione di Fabri Fibra, Giorgio Olmoti e Omar Pedrini.

L'esposizione copre un arco temporale che va dal 1958, vittoria di Modugno a Sanremo con la rivoluzionaria Nel blu dipinto di blu (Volare), al 1982, anno della vittoria dell'Italia al Mondiale di 
calcio, celebrata con un tripudio collettivo che testimonia la rinnovata «voglia di leggerezza» di un popolo dopo un decennio economicamente difficile e profondamente segnato dalla violenza politica.

L'allestimento, curato dalla designer Francesca Seminatore con le installazioni audio-video di Daniele Perrone, è suddiviso in undici aree tematiche ed è una grande rappresentazione della storia italiana attraverso la musica, con una scelta di 100 canzoni capaci di parlare il linguaggio, descrivere $\mathrm{i}$ fatti, e restituire le emozioni dei venticinque anni che hanno profondamente mutato il volto del nostro Paese.[2]

L'Istoreto, per parte sua, ha scelto di svolgere laboratori sulla canzone d'autore in ragione dell'attenzione che rivolge da alcuni anni ai linguaggi della contemporaneità - fra i quali quello della pop music è senz'altro tra i più rilevanti - allo scopo di diffondere nelle scuole una didattica della storia che integri le fonti tradizionali e con quelle non tradizionali.

\section{5 laboratori didattici}

I laboratori dell'Istoreto, svolti nella sede dell'allestimento, sono stati pensati prevalentemente come supporto introduttivo alla visita; in qualche caso, però, sono stati utilizzati come attività di approfondimento a posteriori, grazie alla quale i ragazzi hanno potuto ritornare su singoli aspetti della mostra o riattraversarla virtualmente seguendo il filo d'Arianna di un tema particolarmente rilevante. La definizione dei temi dei laboratori è stata affidata a un gruppo di lavoro composto da ricercatori Istoreto e consulenti esterni (musicologi, didatti), che hanno prodotto una serie di kit composti da materiali utili per le attività con le classi.

Lo svolgimento dei laboratori è stato preceduto da un momento di formazione, rivolto agli insegnanti, che ha riguardato sia i contenuti, sia il metodo didattico.

Per quanto riguarda i contenuti, il gruppo di lavoro ha individuato cinque temi di particolare interesse, ciascuno dei quali ampiamente rappresentato, nelle varie sezioni della mostra, da immagini, canzoni, didascalie, installazioni[3]:

- Le canzoni dei "giovani”

- I consumi

- L'emigrazione

- Le "nuove donne"

- La violenza politica negli anni Settanta

Esaminiamoli velocemente uno per uno, nei loro presupposti storiografici e relativi obiettivi didattici.

\section{Le canzoni dei "giovani"}

A partire dagli anni Cinquanta (in primo luogo negli Stati Uniti) aziende e mercati hanno iniziato a considerare i giovani come destinatari di specifici messaggi esistenziali, politici e commerciali.

Attraverso alcuni brani musicali, gli studenti sono condotti a riflettere su come i giovani, negli anni del «miracolo italiano», siano emersi come una nuova categoria sociale e di consumatori contrapposta, nello stile di vita, alle vecchie generazioni.

\section{I consumi}


Tra gli anni Cinquanta e Sessanta una maggiore disponibilità economica trasforma gli italiani in consumatori. La musica consente di percorrere quegli anni analizzando le grandi trasformazioni sociali in atto che hanno cambiato il Paese.

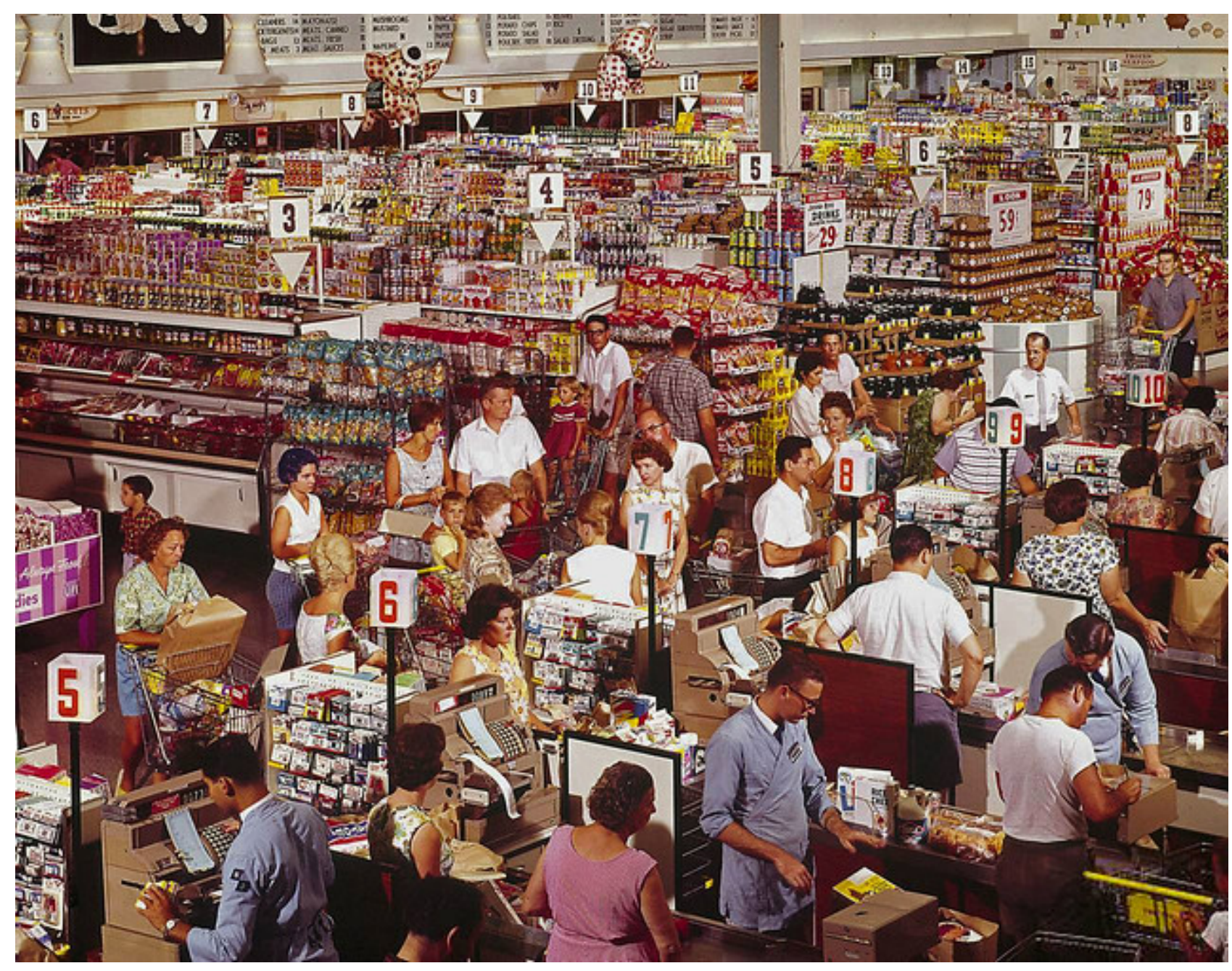

Supermarket negli Usa, 1964

\section{L'emigrazione}

Dalla scelta di alcuni brani musicali, tra quelli che indirettamente e direttamente affrontano il tema dello «spostamento», emerge il quadro di un'Italia in continuo movimento. L'obiettivo è aiutare a comprendere come, negli anni del boom, l'emigrazione abbia cambiato la realtà sociale di tutto il paese.

\section{Le "nuove" donne}

Attraverso alcune canzoni scritte e interpretate da donne, il percorso punta a valorizzare la percezione di sé delle interpreti fino agli anni della rivoluzione sessuale. La canzone viene pertanto analizzata, in questo caso, come strumento per un'autobiografia di genere. 


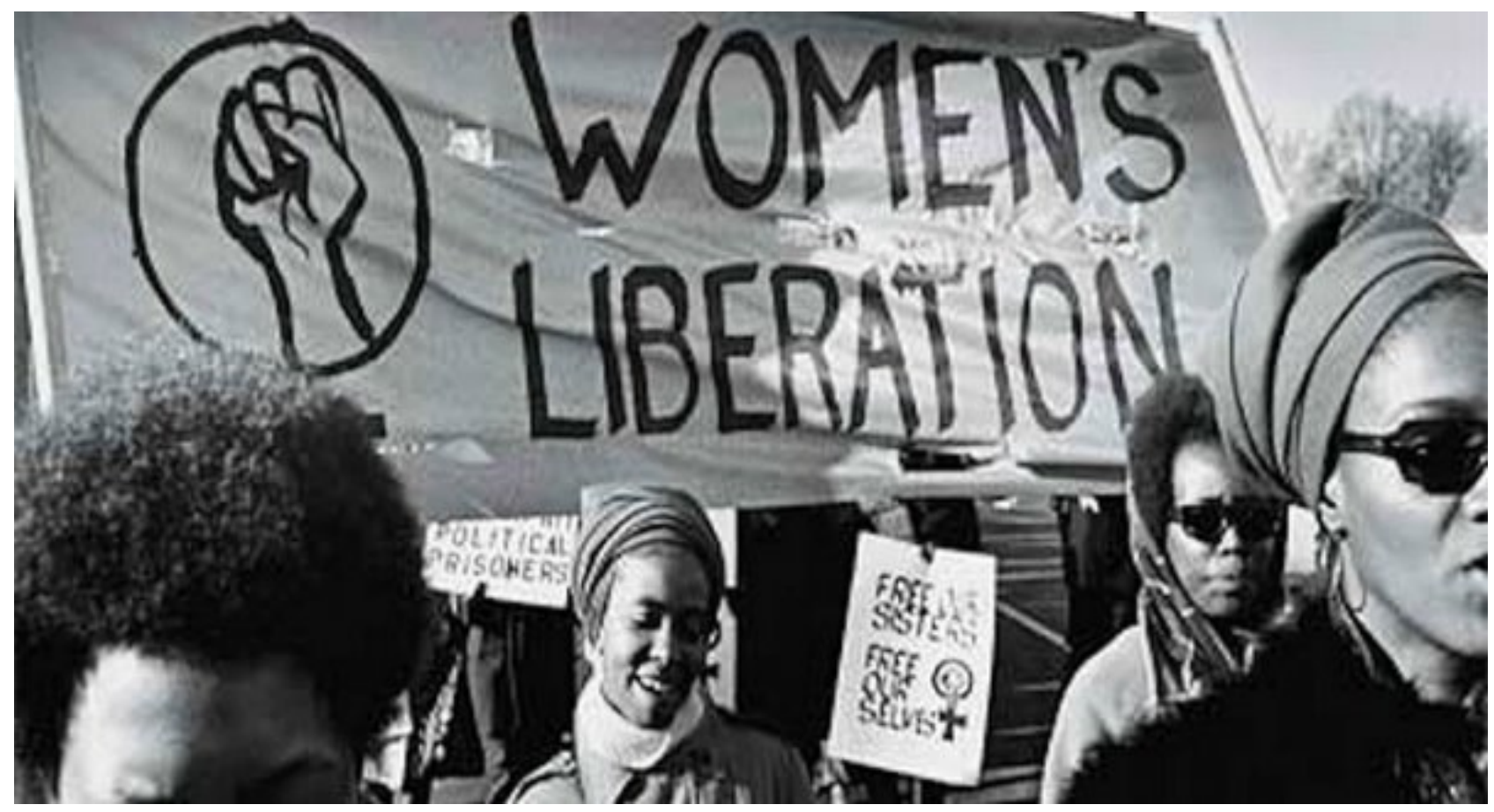

Donne in marcia, 1960 (D. Felton)

\section{La violenza politica}

Le canzoni restituiscono un decennio, quello dei '70, caratterizzato dall' «impegno» e da una dimensione del conflitto politico che contempla l'uso della violenza. L'obiettivo, in questo caso, è analizzare la canzone non tanto come fonte, ma come narrazione e creazione di miti.

I laboratori[4], della durata di 1 ora e 30 minuti, sono stati condotti da due formatori (uno storico e un musicista). Hanno permesso ai ragazzi di accedere in modo partecipato alle conoscenze di base utili a comprendere la mostra, nonché di riflettere sul rapporto tra passato e presente.

L'argomento maggiormente richiesto, com'era facile a prevedersi, è stato quello relativo ai giovani e all'affermazione di una sottocultura giovanile, tema che più di altri si è prestato a gettare ponti fra le diverse generazioni e a suscitare confronti sull'asse della continuità/rottura.

In sede di bilancio, si può dire che i laboratori sulla canzone rappresentino un'importante scommessa per il futuro. Il gruppo di lavoro che ha progettato i percorsi, infatti, si è man mano convinto della loro riproducibilità in forma autonoma (cioè indipendente dal collegamento con la mostra) sia per un pubblico scolastico, sia per un pubblico «adulto»o «misto» avvicinabile attraverso circoli, associazioni e così via presenti sul territorio (periferie, provincia).

\section{La "canzonetta", fonte di grande rilievo}

Qualche considerazione finale in materia di metodologia storica.

Le canzoni e la popular music costituiscono delle fonti spesso non riconosciute nella loro rilevanza. Viceversa, le creazioni musicali (testo e partitura), se adeguatamente interrogate, tramettono informazioni importanti sul contesto in cui sono nate.

Per lo storico infatti, come insegna Giovanni De Luna -tra i curatori della mostra- le canzoni diventano «documenti preziosi per leggere nelle profondità dei gusti e delle emozioni della società 
di massa», a patto però di non guardare ad esse «nei loro aspetti artistici, nei risvolti musicali delle loro melodie e in quelli letterari dei loro testi, ma di declinare il loro rapporto con la storia in almeno tre direzioni diverse...»[5]: come fonti di conoscenza storica; come forme di narrazione di un'epoca; come agenti dei processi storici.

Quest'ultima prospettiva, che indaga l'apporto fornito dalla musica pop in quanto vettore di progresso tecnologico e produttivo, pratica di consumo o strumento di socialità (e costruzione di identità collettiva, generazionale o nazional-popolare), pare oggi foriera di sviluppi particolarmente interessanti. Lo dimostrano, ad esempio, gli interventi di Cesare Grazioli e Ferdinando Fasce pubblicati su questa stessa rivista[6].

\section{Note:}

[1] Per ulteriori informazioni, rinviamo al sito ufficiale http://www.mostranoi.it/, url consultata il 31-12-2019.

[2] La Rai ha mandato in onda un servizio sulla mostra torinese nell'edizione serale del TG1 del 23 marzo 2019. Cfr. http://www.tg1.rai.it/dl/RaiTV/programmi/media/ContentItem-c0bfe0f1-b46d48c5-8cf3-a692e9ec2b95-tg1.html\#p=62 url consultata il 20-11-2019.

[3] Si veda a questo proposito il ricco catalogo della mostra: G. Brusini, G. De Luna, L, Salvini, Noi. Non erano solo canzonette 1958-1982, Skira, Milano 2019.

[4] I laboratori svolti sono stati 15 (per 15 classi), 9 dei quali rivolti ai licei, 1 a un IIS, 4 a istituti tecnici o professionali, 1 a un Istituto Comprensivo, e hanno coinvolto circa circa 300 studenti.

[5] G. De Luna, Quando la musica leggera diventa storia, https://www.lastampa.it/topnews/letteree-idee/2019/03/21/news/quando-la-musica-leggera-diventa-storia-1.33689586.

[6] Di C. Grazioli, 1958-1968. I tanti filoni della musica degli anni '60, la stagione dei giovanissimi; Id, All'inizio del "miracolo economico", due terremoti musicali, entrambi in "Novecento.org", 8, 2017 e Id "Prendi la chitarra e vai/Se ti fermi invecchierai". Quando l'Italia era un paese di giovani e per giovani, in"Novecento.org”, 12, 2019.

Di F. Fasce, ricordiamo il saggio sui concerti italiani dei Fab Four nel 1965 The Beatles in Italy. Per una ricerca sulla storia della popular music e i lunghi anni Sessanta, presentato nel dossier La colonna sonora di una generazione a cura di G. Fulvetti e S. Pivato, in "Novecento.org", 12, 2019, oltre al recente volume La musica nel tempo. Una storia dei Beatles, Einaudi, Torino 2018. 